\title{
An assessment of the importance ofexposure routes to the uptake and internal localisation of fluorescent nanoparticles in zebrafish (Danio rerio), using light sheet microscopy
}

Skjolding, Lars Michael; Ašmonait, G; Jølck, Rasmus Irming; Andresen, Thomas Lars; Selck, H.; Baun, Anders; Sturve, J.

Published in:

Nanotoxicology

Link to article, DOI:

$10.1080 / 17435390.2017 .1306128$

Publication date:

2017

Document Version

Peer reviewed version

Link back to DTU Orbit

Citation (APA):

Skjolding, L. M., Ašmonait, G., Jølck, R. I., Andresen, T. L., Selck, H., Baun, A., \& Sturve, J. (2017). An assessment of the importance ofexposure routes to the uptake and internal localisation of fluorescent nanoparticles in zebrafish (Danio rerio), using light sheet microscopy. Nanotoxicology, 11(3), 351-359. https://doi.org/10.1080/17435390.2017.1306128

\section{General rights}

Copyright and moral rights for the publications made accessible in the public portal are retained by the authors and/or other copyright owners and it is a condition of accessing publications that users recognise and abide by the legal requirements associated with these rights.

- Users may download and print one copy of any publication from the public portal for the purpose of private study or research.

- You may not further distribute the material or use it for any profit-making activity or commercial gain

- You may freely distribute the URL identifying the publication in the public portal 


\title{
Nanotoxicology \\ Nanotoxicology
}

\section{An assessment of the importance ofexposure routes to the uptake and internal localisation of fluorescent nanoparticles in zebrafish (Danio rerio), using light sheet microscopy}

\author{
L. M. Skjolding, G. Ašmonaitè, R. I. Jølck, T. L. Andresen, H. Selck, A. Baun \& J.
} Sturve

To cite this article: L. M. Skjolding, G. Ašmonaitè, R. I. Jølck, T. L. Andresen, H. Selck, A. Baun \& J. Sturve (2017): An assessment of the importance ofexposure routes to the uptake and internal localisation of fluorescent nanoparticles in zebrafish (Danio rerio), using light sheet microscopy, Nanotoxicology, DOI: 10.1080/17435390.2017.1306128

To link to this article: http://dx.doi.org/10.1080/17435390.2017.1306128

Accepted author version posted online: 11

Mar 2017.

Submit your article to this journal $₫$

Щ Article views: 13

Q View related articles ¿

View Crossmark data $\nearrow$ 


\section{An assessment of the importance ofexposure routes to the uptake and internal localisation of fluorescent nanoparticles in zebrafish (Danio rerio), using light sheet microscopy}

*Skjolding L. M. ${ }^{1}$, Ašmonaitė G. ${ }^{2}$ Jølck R. I. ${ }^{3}$, Andresen T. L. ${ }^{3}$, Selck H. ${ }^{4}$, Baun A. ${ }^{1}$ and Sturve J. ${ }^{2}$ ${ }^{1}$ Department of Environmental Engineering, Technical University of Denmark, Building 113, DK2800 Kgs. Lyngby, Denmark

${ }^{2}$ Department of Biological and Environmental Sciences, University of Gothenburg, Medicinaregatan 18A, SE-41390 Gothenburg, Sweden

${ }^{3}$ Department of Micro- and Nanotechnology, Center for Nanomedicine and Theranostics, Technical University of Denmark, Building 423, DK-2800 Kgs. Lyngby, Denmark

${ }^{4}$ Department of Science and Environment, Roskilde University, DK-4000 Roskilde, Denmark

*Corresponding author: lams@env.dtu.dk

Phone: +45 45251477

Address: DTU Environment

Miljøvej, Building 113

DK-2800 Kgs. Lyngby 


\begin{abstract}
(249 words)
A major challenge in nanoecotoxicology is finding suitable methods to determine the uptake and localisation of nanoparticles on a whole-organism level. Some uptake methods have been associated with artefacts induced by sample preparation, including staining for electron microscopy. This study used light sheet microscopy (LSM) to define the uptake and localisation of fluorescently labelled nanoparticles in living organisms with minimal sample preparation. Zebrafish (Danio rerio) were exposed to fluorescent gold nanoparticles (Au NPs) and fluorescent polystyrene NPs via aqueous or dietary exposure. The in vivo uptake and localisation of NPs was investigated using LSM at different time points (1, 3 and 7 days). A time-dependent increase in fluorescence was observed in the gut after dietary exposure to both Au NPs and polystyrene NPs. No fluorescence was observed within gut epithelia regardless of the NP exposure route indicating no or limited uptake via intestinal villi. Fish exposed to polystyrene NPs through the aqueous phase emitted fluorescence signals from the gills and intestine. Fluorescence was also detected in the head region of the fish after aqueous exposure to polystyrene NPs. This was not observed for Au NPs. Aqueous exposure to $\mathrm{Au}$ NPs resulted in increased relative swimming distance, while no effect was observed for other exposures. This study supports that the route of exposure is essential for the uptake and subsequent localisation of nanoparticles in zebrafish. Furthermore, it demonstrates that the localisation of NPs in whole living organisms can be visualised in real-time, using LSM.
\end{abstract}




\section{Introduction}

Nanoparticles (NPs) are used in a wide range of industrial applications as well as in consumer products, thereby making inevitable the release of NPs into the environment. Even though limited information exists about their fate and persistence in the aquatic environment (Peijnenburg et al. 2015), evidence is building that indicates NPs are taken up by aquatic organisms at different trophic levels (Hoolbrook et al. 2008; Cleveland et al. 2012; Hou et al. 2013; Khan et al. 2015). Trophic transfer has been proposed as an important process for NP uptake in fish (Cedervall et al. 2012; Geffroy et al. 2012; Ladhar et al. 2014; Skjolding et al. 2014; Batel et al. 2015; Mattsson et al. 2015), and furthermore it could be suggested that the internal transport, localisation and subsequent toxicity of NPs may differ for organisms through aqueous and dietary exposure. In general, there is a lack of information on the extent of NP uptake at the organism level, and recent concern has been raised regarding the relationship between the uptake route and internal localisation of NPs both in vivo and in vitro (Bondarenko et al. 2013; Fabrega et al. 2011; Ma et al. 2013; Menard et al. 2011; Scown et al. 2010; Tourinho et al. 2012; Xin et al. 2014). The influence of aqueous or dietary NP exposure has been examined in fish (Ates et al. 2014; Skjolding et al. 2014) and aquatic invertebrates (Croteau et al. 2014; Larguinho et al. 2014; Ramskov et al. 2015; Dai et al. 2015); however, knowledge regarding the link between exposure route and localisation is still somewhat limited (Croteau 2014; Ramskov et al. 2015).

The techniques available for experimentally determining NPs in organism tissues place serious limits on the qualitative and quantitative analyses of uptake and internal localisation. Light sheet microscopy (LSM) is a new and promising technique that offers the potential to be used as a complement to the widely used electron microscopy. The major advantage of LSM is that living organisms can be imaged in real time in a non-invasive manner, thus retaining the integrity of the 
test organism, which in electron microscopy would undoubtedly be lost. Furthermore, a low level of invasiveness in relation to embedding and imaging, good penetration depth, low photo-bleaching and simple sample preparation, compared to other types of microscopy, make this technology highly attractive for the in situ visualisation of NPs in living organisms.

Locomotion is a pivotal function of and the basis for all animal behaviours. Altered swimming performance can serve as a sensitive and integral bioindicator of neurotoxicity and provide valuable information on the sensory-motor function/perception of exposed animals. Indeed, studies have shown increased levels of NPs in the brains of fish after aqueous exposure (Kashiwada 2006; Asharani et al. 2008; Ramsden et al. 2009; Zhu et al. 2010). The uptake of and translocation to the brain through the olfactory tract have been proposed in this regard (Handy et al. 2008; Shaw and Handy, 2011), and there is evidence that engineered metal NPs can enter brain tissues and act as neurotoxicants, subsequently causing sensory-motoric dysfunction. It has also been shown that silver nanoparticles cause behavioural alterations in developing zebrafish larvae (Powers et al. 2011; Ašmonaitė et al. 2016), whereas exposure to Au NPs in the early life stages of zebrafish result in abnormal swimming behaviour, persisting into adulthood (Truong et al. 2012).

This study aimed at investigating the influence of exposure routes on 1) the uptake, internal NP localisation and potential behavioural effects of two types of nanoparticles in zebrafish and 2) the feasibility of using LSM in the context of ecotoxicological tests, which to our knowledge has not been done previously. Fluorescent polystyrene NPs were used as a reference material for assessing uptake and localisation while using the novel LSM technique. Furthermore, the polystyrene NPs represents a model particle both in nanoparticle and microplastics research. Fluorescent gold nanoparticles (Au NPs) were used to assess both uptake and localisation using LSM, and to quantify uptake through inductively coupled plasma mass spectrometry (ICP-MS). This 
combination of a qualitative (LSM) and a quantitative (ICP-MS) method was used to improve our understanding of the importance of uptake route (aqueous vs dietary (Artemia salina)) for uptake and localisation in fish, and for the direct validation of LSM as a functional tool for studying NPs in living organisms. Zebrafish behaviour assessment was conducted to complement LSM and massquantification results by indirectly assessing the toxicity on sensory-motor function, using locomotion as a proxy for behavioural alterations.

\section{Materials and Methods}

\subsection{Chemicals and solutions}

Fluorescent carboxylate-modified polystyrene nanoparticles (FluoroSpheres ${ }^{\circledR}, 2 \%$ solids) with a reported size of $20 \mathrm{~nm}$ were purchased from Invitrogen (Oregon, USA). Au NPs with a fluorescein probe were prepared with slight modifications according to the procedure provided by Jølck et al. (2015) and described in further detail below. The zebrafish embryo medium consisted of $\mathrm{MgSO}_{4} * 7 \mathrm{H}_{2} \mathrm{O} 245 \mathrm{mg} / \mathrm{L}, \mathrm{KH}_{2} \mathrm{PO}_{4} 20.5 \mathrm{mg} / \mathrm{L}, \mathrm{Na}_{2} \mathrm{HPO}_{4} 6 \mathrm{mg} / \mathrm{L}, \mathrm{CaCl}_{2} * 2 \mathrm{H}_{2} \mathrm{O} 145 \mathrm{mg} / \mathrm{L}, \mathrm{KCl} 37.5$ $\mathrm{mg} / \mathrm{L}$ and $\mathrm{NaCl} 875 \mathrm{mg} / \mathrm{L}$ dissolved in MilliQ water. Artificial saltwater for the brine shrimp culture was prepared using commercial salts (Instant Ocean, Aquarium Systems, FR).

\subsection{Au NPs-fluorescein synthesis}

All glassware was cleaned in freshly prepared aqua regia, rinsed thoroughly with MilliQ water $(18.2 \mathrm{M} \Omega \cdot \mathrm{cm})$ and then dried at $150^{\circ} \mathrm{C}$ before use. An aqueous solution of $\mathrm{HAuCl}_{4} \cdot 4 \mathrm{H}_{2} \mathrm{O}(0.23 \mathrm{mM}$, $220.4 \mathrm{~mL})$ was adjusted to $\mathrm{pH} 7.0$ by the dropwise addition of $\mathrm{NaOH}(0.10 \mathrm{M})$. Sodium acrylate (40.9 mM, 73.5 mL) was added and the reaction flask gently swirled by hand. The reaction mixture was left at room temperature, without stirring, for 5 days. The formed acrylate stabilised Au NPs were PEGylated using ( 8 polyethylene plycol (PEG) molecules $/ \mathrm{nm}^{2}$ ) by adding an aqueous 99:1 
mixture of $\mathrm{MeO}-\mathrm{PEG}_{5.000}-\mathrm{SH}(6.72 \mathrm{mg}, 1.34 \mu \mathrm{mol})$ and fluorescein-PEG ${ }_{5.000}-\mathrm{SH}(67.86 \mu \mathrm{g}$, $13.57 \mathrm{nmol})$. The mixture was stirred for $24 \mathrm{~h}$ at room temperature and protected from sunlight. The fluorescein-labelled PEGylated Au NPs were purified by centrifugation (9.500 RPM, 20 min/cycle), extensively washed with MilliQ water (×3) and up-concentrated to $1000 \mu \mathrm{g} \mathrm{Au} / \mathrm{mL}$.

\subsection{Nanoparticle characterisation}

The size and stability of Au NPs and polystyrene NPs in the exposure medium were characterised by dynamic light scattering (DLS) (Malvern, Zetasizer Nano-ZS, UK). To determine size and zetapotential, fresh stock solutions were prepared prior to DLS measurements. No sonication or prolonged stirring was used. For Au NPs and polystyrene NPs, concentrations of $1 \mathrm{mg} \mathrm{Au} / \mathrm{L}$ and 5 $\mathrm{mg}$ polystyrene/L in zebrafish embryo media were used, respectively. Each measurement was performed at $20^{\circ} \mathrm{C}$ in triplicate, with 10 runs of a $1 \mathrm{~mL}$ sample. A backscattering angle of $173^{\circ}$ was used. The Stokes-Einstein equation was used to calculate the hydrodynamic diameter of the NPs (Au and polystyrene), using the cumulant method to fit the autocorrelation function (Kretzschmar et al., 1998).

\subsection{Zebrafish and brine shrimp husbandry}

The transparent (pigmentless) mutant line Casper Zebrafish ${ }^{\mathrm{TM}}$ was selected for this study, because a relatively high light dispersion in the tissues ensures better spatial resolution compared with other wild-type zebrafish strains. Zebrafish were kept and bred at the Institute of Neurosciences and Physiology, University of Gothenburg, and the embryos were hatched and reared at the Department of Biological and Environmental Sciences, University of Gothenburg. Zebrafish were kept in a temperature-controlled room at $25^{\circ} \mathrm{C}$ with a $14 \mathrm{~h} / 10 \mathrm{~h}$ light/dark cycle in zebrafish embryo medium and were fed twice a day with newly hatched brine shrimp nauplii which were hatched 
approximately $24 \mathrm{~h}$ prior to use in artificial $20 \%$ saltwater under constant aereation. Juvenile zebrafish: (4-week-old zebrafish for Au NP exposure and 6 weeks for polystyrene NPs) were used for the experiments (different stages of juvenile zebrafish were sued due to time constraints on the microscope, it was not deemed to influence the results of the study).

\subsection{Exposure scenarios for aqueous and dietary exposure of zebrafish}

All experiments ran for 7 days and were conducted in $1.5 \mathrm{~L}$ plastic aquaria $(n=7$, for each type of NP tested: three aqueous exposures, three dietary exposures and one control) filled with $1 \mathrm{~L}$ zebrafish embryo medium and accommodating three juvenile fish. For aqueous exposure, $5 \mathrm{mg}$ polystyrene/L of polystyrene NPs, or $1 \mathrm{mg} \mathrm{Au} / \mathrm{L}$ of $\mathrm{Au}$ NPs, were prepared in the zebrafish embryo medium. Daily, un-exposed brine shrimp nauplii were administered as a food source for aqueousexposed fish. Dietary exposure was carried out with newly hatched brine shrimp exposed to $5 \mathrm{mg}$

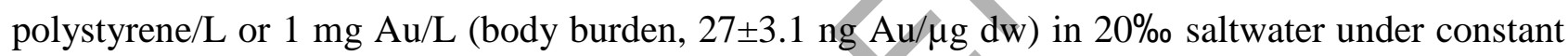
aeration for $24 \mathrm{~h}$. Concentrations were selected based on previous studies with Daphnia magna (for $\mathrm{Au}$ NPs) and pilot studies with the polystyrene NPs. In addition, the aqueous exposure concentration was set equally between zebrafish exposure and brine shrimp exposure, to allow for the realistic dietary exposure of zebrafish. Prior to feeding, brine shrimp were rinsed in zebrafish embryo medium to remove loosely bound NPs and exposure solution before feeding. For dietary exposures, juvenile zebrafish were kept in uncontaminated zebrafish embryo medium and fed $a d$ libitum once a day with pre-exposed brine shrimp nauplii (body burden, $27 \pm 3.1 \mathrm{ng} \mathrm{Au} / \mu \mathrm{g} \mathrm{dw}$ for Au NPs). Ad libitum food was administered to minimise differences caused by competitive feeding patterns and trends of larger zebrafish eating all the available food. Leftover food was removed prior to the addition of new brine shrimp. Unexposed fish $(n=3)$, reared under similar conditions, were included as controls. Exposed zebrafish from one aquarium were collected and imaged by 
LSM after 1, 3 and 7 days with either dietary ( $n=3$, per time point) or aqueous exposure ( $\mathrm{n}=3$, per time point), as detailed in the following procedures described below. All experimental protocols were approved by an ethical committee on animal experimentation (permit number: 274-2011).

\subsection{Sample preparation for light sheet microscopy}

Prior to LSM measurement, the zebrafish were transferred briefly to a clean embryo medium, to be rinsed of exteriorly bound NPs. For anaesthesia, a $0.55 \mathrm{mg} / \mathrm{L}$ phenoxyethanol solution in a zebrafish embryo medium was used. After anaesthesia the test organism ( $n=3$ per time point) was embedded in freshly prepared $1 \%$ low-temperature melting agarose and kept on a heating block at $38^{\circ} \mathrm{C}$ to keep it liquefied. Subsequently, individual zebrafish were carefully transferred into an Eppendorf tube containing this agarose solution. An appropriate glass capillary with a plunger was used to enclose individual zebrafish in the confined agarose bed. During this procedure, anchored specimens were carefully aligned along the vertical axis of the capillary. This ensured the best light penetration from all angles when imaged and allowed for better comparison between different samples. Once the agarose had solidified (after approximately $1 \mathrm{~min}$ ) the sample was placed in an LSM chamber for imaging (Reynaud et al., 2015). During imaging the zebrafish were still alive, visualized by blood flow and heartbeat.

\subsection{Settings used for light sheet microscopy and image analysis}

The LSM system (Lightsheet Z.1, Carl Zeiss Microscopy, DE), equipped with a $\times 5$ objective, was used for imaging embedded zebrafish. Standard exposure chambers were filled with zebrafish embryo media, and no anaesthetics were added. Samples were excited with two lasers, the first (488 $\mathrm{nm})$ capturing autofluorescence and the second $(566 \mathrm{~nm})$ used to excite fluorophores, thereby yielding the fluorescence signal of the NPs. A laser intensity of 3\% was used to acquire 
autofluorescence (broad pass filter 505-545 nm) and depict the outline of the zebrafish (and was used as a reference). Different properties of the second laser were applied for imaging Au NPs and polystyrene NPs. When imaging polystyrene NPs, a 575-615 nm broad pass filter was used and the laser intensity was $10 \%$, whereas for Au NPs, a broad pass filter of 505-545 nm was used for excitation and the laser intensity was $30 \%$. This was done to accommodate the emission wavelength of the surface-bound fluorescein-fluorophores. The exposure time used for all the samples was $154.8 \mathrm{~ms}$. Imaging was performed with light directed from both sides, thereby yielding maximum sample illumination and avoiding shaded areas that may have been caused by illuminating from just one side. All imaging was performed as a series of slides, referred to as the "z-stack," using an optimal slide thickness determined by ZEN software (Carl Zeiss Microscopy, DE). A 3D-multiview of the sample was made by rotating samples at six different angles $\left(0^{\circ}, 60^{\circ}, 120^{\circ}, 180^{\circ}, 240^{\circ}, 300^{\circ}\right.$ and $360^{\circ}$ ) and fusing them with a pixel average of 3 in the $x$ - and y-directions. Maximum intensity projection was created to evaluate the overall uptake of Au NPs and polystyrene NPs.

\subsection{Behavioural toxicity assessment}

Locomotion-based behavioural properties of differentially exposed fish were used as endpoints to investigate the potential biological effects of $\mathrm{Au}$ NPs and polystyrene NPs. The swimming behaviour (locomotion) of juvenile zebrafish was determined by using the View Point ${ }^{\circledR}$ Video tracking system (VideoTrack V3, ViewPoint Life Science Inc, Montreal, CAN). A customised protocol, composed of 10 sequentially interchanging dark-light cycles, was used to artificially stress fish and evoke visual-motor responses, as described by Ašmonaitè et al. (2016). The behavioural experiments started with $30 \mathrm{~min}$ light acclimatisation, followed by interchanging cycles of $10 \mathrm{~min}$ darkness and then followed by 5 minutes of light stimulation (with $100 \%$ light saturation) (Ašmonaitė et al. 2016). 
The locomotion of zebrafish exposed to Au NPs or polystyrene NPs after aqueous and dietary exposures (after 1, 3 and 7 days) was studied. All experiments were performed in six well-plates, each containing a $5 \mathrm{~mL}$ treatment $(1 \mathrm{mg} \mathrm{Au} / \mathrm{L}$ as $\mathrm{Au}$ NPs or $5 \mathrm{mg}$ polystyrene/L as polystyrene NPs)/control suspension and one juvenile fish. The movement tracking of individuals was registered simultaneously at a rate of 25 images per second (according to CCIR standards), and the integration time for data was 10 seconds. The behavioural experiments were conducted consistently between 12:00 and 16:00 h.

\subsection{Chemical analysis}

Prior to chemical analysis, whole-fish samples were freeze-dried and digested in $2 \mathrm{~mL}$ aqua regia (3:1 HCl:HNO $3 \mathrm{v} / \mathrm{v})$ at room temperature for at least $24 \mathrm{~h}$ in the dark. During digestion, no heat or other additional treatment was applied. Prior to chemical analysis, $8 \mathrm{~mL}$ of distilled water was added and the samples were decanted into disposable plastic vials. Chemical analysis was carried out using ICP-MS (7700x, Agilent Technologies) with an internal yttrium standard.

\subsection{Statistical analysis}

All uptake experiments were carried out in triplicate, and for each dataset the mean and standard deviation (SD) was calculated. Mean values for the uptake and characterisation experiments were recorded as mean $\pm \mathrm{SD}$ (figure 3). For behavioural data, values were reported as mean $\pm \mathrm{SE}$ (figure 4) with four replicates. All datasets were tested for the normality and homogeneity of variance before statistical analysis. For the analysis of uptake data, the data were log-transformed for equal variance, followed by a one-way ANOVA. Tukey's pairwise comparison test was used to test for significant differences among all concentrations, and Dunett's test was used to test exposures 
against the control (GraphPad Prism v5.0). Datasets were considered statistically significantly different at a $\mathrm{p}$ value $<0.05$.

\section{Results}

\subsection{Characterisation of nanoparticles}

Initial measurements $(\mathrm{t}=0)$ showed a $\mathrm{z}$-average of $71 \pm 4 \mathrm{~nm}$ for $\mathrm{Au}$ NPs and $610 \pm 130 \mathrm{~nm}$ for polystyrene NPs in zebrafish embryo medium (Table 1). A polydispersity index (PDI) of $0.21 \pm 0.08$ was observed for the Au NPs, while the polystyrene NPs showed a higher initial PDI of 0.44 \pm 0.05 . After 24 hours of incubation in zebrafish embryo medium, the hydrodynamic diameter of the $\mathrm{Au}$ NPs increased to $89 \pm 21 \mathrm{~nm}$, with a concurrent increment in PDI to $0.27 \pm 0.03$. Au NP size distribution was reported as a single uniform peak and corresponded with the relatively low PDI (Table 1). Polystyrene NPs increased markedly in hydrodynamic diameter to $3580 \pm 410 \mathrm{~nm}$ during the $24 \mathrm{~h}$ incubation period. Concurrently, the PDI increased to 1 , thus indicating a highly polydispersed sample. For the polystyrene NPs, information reported from the DLS measurements in terms of size should therefore only be used to interpret trends in agglomeration and not exact hydrodynamic size.

\subsection{Aqueous and dietary exposure of fluorescent polystyrene nanoparticles to zebrafish}

The analysis of the LSM images is a subjective assessment of the fluorescent signal based on three fish per time point, for which representative images for each time point are shown herein (Figures 1 and 2).

After the first day of aqueous exposure to polystyrene NPs, an increased fluorescent signal was observed throughout the whole body of the exposed zebrafish (Figure 1a) compared to unexposed control animals (Figure 1g). More intense fluorescence was observed in the head and gut regions, 
presumably due to higher polystyrene NP accumulation in these segments of the fish (Figure 1a). The sorption of polystyrene NPs in gill arches and pectoral fins was observed (Figure 1a). After three days of aqueous exposure the signal from the gut and head regions decreased (Figure 1b). Fluorescence was still observed in the branchial arches and the olfactory area, and a fine fluorescent signal outlined the fins. After seven days of aqueous exposure the fluorescent signal in the head regions was less pronounced (Figure 1c). A readily scattered signal remained in the head region and the olfactory tract. A strong fluorescence signal, compared to the control, was depicted in the gut region and was associated with round structures collected in the intestinal bulb (Figure 1c).

For dietary exposure to polystyrene NPs a clear fluorescent signal was confined to the gastrointestinal region for all tested time points, though there was a tendency to increase at day 7 (Figure 1d-f): after the first day of exposure, a stronger fluorescent signal from the posterior intestine was observed (Figure 1d), after the third day more fluorescence was emitted from the intestinal bulb (Figure 1e) and after seven days the overall fluorescence signal was stronger and diffused throughout the gut and gill compartments (Figure 1f). Throughout the dietary exposure period (1-7 days) no elevated fluorescent signal was observed outside the gut region.

\subsection{Aqueous and dietary exposure of Au NP to zebrafish}

Only a very weak fluorescent signal was recorded for zebrafish exposed to Au NPs through the aqueous phase, as the signal in exposed zebrafish increased only slightly compared to the control zebrafish (Figure 2a-c). After three days of aqueous exposure a weak fluorescence signal was observed from the gut region (Figure $2 \mathrm{~b}$ ), but this was not found again when examining after seven days of exposure (Figure 2c). During dietary exposure a clear fluorescent signal arising from Au NPs was visible and restricted to the gut region at all three time points (Figure 2d-f). There was no 
clear indication of a change in signal over time. Fluorescence clearly outlined macrostructures in the gut region of the zebrafish.

The uptake of Au NPs in zebrafish tissue was quantified using ICP-MS (detection limit, $0.09 \mu \mathrm{g}$ $\mathrm{Au} / \mathrm{L})$ and is shown in Figure 3 as a function of exposure time. The body burden of $\mathrm{Au}$ NPs in zebrafish exposed to Au NPs through aqueous exposure was higher than for the unexposed control group (background concentration $0.051 \pm 0.012 \mu \mathrm{g} \mathrm{Au} / \mathrm{L})(\mathrm{p}<0.05)$ at all time points, and it remained at a constant level throughout the testing period $(\mathrm{p}>0.05)$ (Figure $3 \mathrm{a})$. The average $(\mathrm{n}=3)$ body burden (day 1-7) was $1.42 \pm 0.96 \mathrm{ng} \mathrm{Au} / \mathrm{mg} \mathrm{dw}$ tissue following aqueous exposure. For dietary exposure (Figure 3b) a linear increase in Au body burden in zebrafish was obseryed over the course of the exposure period, with all points being statistically significantly different from the control group ( $\mathrm{p}<0.05$ ). After one day of exposure the body burden was $1.47 \pm 0.76 \mathrm{ng} \mathrm{Au} / \mathrm{mg} \mathrm{dw}$ tissue $(\mathrm{n}=3)$, which increased $\times 8.6$ at day three $(12.7 \pm 2.5 \mathrm{ng} \mathrm{Au} / \mathrm{mg} \mathrm{dw}, \mathrm{n}=3)$ and $\times 26$ at day seven (38.3 $\pm 7.8 \mathrm{ng} \mathrm{Au} / \mathrm{mg} \mathrm{dw}, \mathrm{n}=3$ ) compared to day 1 .

\subsection{Behaviour toxicity assessment}

A trend of increasing relative swimming activity was observed for zebrafish exposed to Au NP through the aqueous phase after 3 and 7 days of exposure; however, there was no significant change in swimming behaviour from day 1 to day $7(\mathrm{p}<0.05)$ (Figure $4 \mathrm{a})$. In contrast, fish exposed to aqueous polystyrene NPs had the highest locomotive response on the first day, whereas the activity decreased over time; however, the decrease was not statistically significant (Figure 4b). Dietary exposure did not result in a consistent time-dependent trend for either Au NPs or for polystyrene NPs (Figure 4), and no statistically significant difference was observed within or between the two types of NPs. 


\section{Discussion}

\subsection{Aqueous and dietary exposure to nanoparticles in zebrafish}

In this study, stomach and intestinal tracts were observed to be important accumulation sites for both particle types (Au NPs and polystyrene NPs) as well as for both exposure routes (Figure 1 and 2), while the intestinal tract appeared more important to dietary exposure. The fluorescent signal following aqueous exposure seemed to decrease over time (Figure 1a-c), possibly due to a decrease in the aqueous phase concentration of polystyrene NPs. This corresponds with the observed dramatic increase in particle size over time, which likely will have caused sedimentation and thus reduce the polystyrene NPs in the water phase (Table 1). Conversely, zebrafish exposed through diet increased the fluorescent signal over time, independent of NP type, thereby indicating that exposure concentration was more constant through this form of exposure (i.e. continuous feeding with pre-exposed brine shrimp). Dietary exposure of pre-exposed brine shrimp to zebrafish demonstrated the transfer of nanoparticles in a simplified aquatic food chain, as both Au NPs and polystyrene NPs administrated through diet were deposited in the stomach and intestines of zebrafish. However, it should be noted that ingestion of NPs from the aqueous phase could happen through gulping behavior during the aqueous exposure, which partly would explain the signal from the intestine during the aqueous exposure.

It has been previously demonstrated that fluorescent polyethylene particles $(1-5 \mu \mathrm{m})$ can pass mucus layers and adhere to intestinal villi in adult zebrafish and that these micro-sized particles may consequently be taken up by endothelial cells (Batel et al., 2015). Merrifield et al. (2013) reported that ingested $\mathrm{Cu}$ NPs were shown to reduce the level of beneficial gut bacteria in fish, with no clear evidence of $\mathrm{Cu}$ NPs being absorbed over gut epithelial membranes. This raises the question as to whether NPs will be retained in the stomach/intestine or taken up and distributed in organism tissue. In our study, no detectable signal was observed in regions other than gut lumen for both NPs 
tested through dietary exposure. This indicates the absence of or the very limited NP uptake through gut epithelia, possibly because the translocation of polystyrene NPs and Au NPs could be below LSM detection limits or could have been minimal within the time frame used in this study. On the other hand, the uptake of different-sized Au NPs exposed through the diet (shrimp flour paste) in zebrafish was studied by Geffroy et al. (2012) over a period of 60 days. The study found limited uptake in brain, liver and skeletal muscle regardless of NP size, albeit not in all exposed zebrafish, thus indicating that uptake could be very limited or possibly associated with other stressors. Conversely, Dedeh et al. (2015) found no quantifiable uptake of Au NPs in zebrafish brains and muscle after 20 days of exposure to $\mathrm{Au}$ NP-contaminated sediment. We found that uptake, measured as fluorescence, only deviated from the control in the gills and the digestive tract. Zhu et al. (2010) found elevated levels of Au NPs following aqueous exposure (0.8-1 mg/L) in brain, heart, liver, gills, dorsal fins and intestines after 24 hours of exposure. In the present study no signal was observed in brains or livers after dietary or aqueous exposure to Au NPs. However, it should be noted that with possible sorption or precipitation, overall concentration in the exposure medium could be decreased in the present study. Furthermore, the detection limit of the fluorescent method could be below that of the ICP-MS method employed by Zhu et al. (2010). Following dietary exposure, the intestinal regions were highly fluorescent, in accordance with findings from Dedeh et al. (2015) and Zhu et al.(2010).

In contrast to dietary exposure, gills and intestines were identified as primary uptake sites for aqueous polystyrene NP exposure. Similar observations were made by Kashiwada (2006), who found increased concentrations of latex beads in different compartments (brain, gills, liver, kidney, gallbladder and intestine) of a transparent medaka (Oryzias latipes) after 7 days of aqueous exposure to $39.4 \mathrm{~nm}$ particles (10 $\mathrm{mg} / \mathrm{L})$. We assessed uptake based on the emission signal, which 
makes comparison between differently fluorescent-labelled NPs difficult. Thus, the seemingly higher uptake of polystyrene NPs compared to Au NPs could be related to a stronger emission signal. Another possibility could be due to the perturbation of lipid membranes caused by the polystyrene NPs. Rossi et al. (2013) observed this perturbation effect in an in vitro model system, using hydrophobic polystyrene chains aggregated to up to $7 \mathrm{~nm}$. However, it should be noted that the polystyrene NPs used by Rossi et al. (2013) were markedly smaller than the majority of the polystyrene NPs used in this study. Furthermore, it should be noted that the hydrophilic PEG-coated $\mathrm{Au}$ NPs would naturally have a lower potential for penetration compared to the hydrophobic polystyrene NPs used by Rossi et al. (2013).

Polystyrene NPs associated with a protein corona has also been shown to affect fat metabolism in Crucian carp (Carassius carassius) following uptake (Cedervall et al., 2012). Consequently, in an environmental setting with exposure through multiple routes, differences in both internal localisation and effect could be observed. However, generalisation on causes and effects is troublesome, due to conflicting evidence existing in the literature regarding NP uptake and tissue distribution, possibly due to the tremendous variations in particle size, surface charge, coating method, exposure route and exposure level.

Overall, the use of LSM enabled a comprehensive qualitative overview of NP uptake and localisation routes by tracking nanoparticles tagged with a fluorescent probe. Furthermore, using image programs to quantify the fluorescence could assist comparison between studies and in studies where a long series of images would not be practical. Generally, a lower fluorescence signal was registered for Au NPs compared with polystyrene NPs. First, the lower signal could be due to potential quenching by $\mathrm{Au}$ NPs. Second, differences in labelling percentages could be a confounding factor. However, the labelling percentage for the polystyrene NPs was not reported by 
the supplier. Lastly, the five-fold higher exposure to polystyrene NPs could have led to a higher uptake, resulting in an increased fluorescent signal. Rothen-Ruthishauser et al. (2014) found a signal limit of approximately $150 \mathrm{Au}$ NPs clusters as the lower boundary. Thus, it is possible that even though no clear signal was observed for Au NPs in this study, diffuse uptake across the gut lumen could be too low for detection with the present technique. The fluorescein probe attached to Au NPs had broad emission spectra, which partially overlapped with the autofluorescence of zebrafish. This feature hampered the distinction of the luminous signal relating to NP uptake in the fish organism. The selection of a probe with narrower emission spectra could improve LSM outcomes and should therefore be considered for future studies. However, the method is generally applicable to organisms with a good light penetration. Fluorescent probe detachment following the elimination of Au NPs is possible; therefore, quantifying uptake through element analysis was necessary to confirm the presence of NPs in the test organisms. Using ICP-MS, Au NP accumulation in zebrafish was indeed confirmed. All presented measurements showed Au content significantly different from that of the control while also showing a florescent signal in the gut lumen of the dietary-exposed fish. Moreover, accumulated Au levels following the dietary exposure quantified in this study exceeded that found by Geffroy et al. (2012), most likely due to the higher dietary administered dose (106 ng Au/fish/day in Geffroy et al. (2012)). It is clear from our findings that the route of exposure had a marked impact on the uptake and localisation of NPs. However, care should be taken when considering the possible decreasing concentration of NPs in aqueous exposures. In addition, we recommend developing the dietary exposure setup further by, for example, controlling exposure: directly administering contaminated food to the test organisms thus providing a means to know exactly how much the fish ingest and minimising loss (i.e. uneaten food items) to the test vessel and sedimentation and thus potential dissolution. Furthermore, the difference in uptake and internal localisation could possibly lead to different toxic mechanisms in 
the test organism, and so it is clear that testing multiple exposure routes would be necessary to cover different aspects of toxic mechanisms.

\subsection{Behavioural toxicity}

The present study offered a unique combination of methods with which to study simultaneously uptake and localisation in fish as well as the potential effects on behaviour of NPs via two exposure routes in transparent zebrafish. It was shown that zebrafish are not only a suitable model for LSM microscopy, but they also represent a well-known model organism in behavioural research (Gerlai, 2011). The observed high fluorescence in the head region indicates transfer to the brain, which may lead subsequently to behavioural toxicity in exposed zebrafish. It has previously been documented that nanoparticles can pass the blood-brain barrier and enter the brains of fish $(25 \mathrm{mg} / \mathrm{L})$ (Asharani et al., 2008), potentially causing neurotoxic effects (Powers et al., 2011; Xin et al., 2015). There is also evidence in the literature that polystyrene (130 mg per feeding) and Au nanoparticles (50 mg/L) can alter fish behaviour (Cedervall et al., 2012; Mattsson et al., 2014; Truong et al., 2012). However, the locomotion assessment carried out in this study did not show any clear changes in visual-motor responses in exposed fish. A tendency towards increased locomotion during a light/dark challenge test for zebrafish exposed to aqueous Au NPs was notified, though the increase in swimming activity may be related to anxiety-like behaviours (Kalueff et al., 2013). It should also be noted that zebrafish juveniles generally show lower responsiveness to artificial light/dark stimulation compared to zebrafish larvae, for which the given behavioural protocol was optimised. Thus, the effectiveness of light stimulation for testing the integrity of sensory-motoric functions for postlarval stages may be limited. While the changes in swimming patterns were not significant, it should be noted that the interpretation and validation of automatic behaviour (locomotion) tracking tool data is a general issue that needs to be studied further (Kalueff et al., 2013; Kane et al., 2005; 
Scott, Sloman et al., 2004). One important consideration regarding behaviour study relates to the considerably small number $(n=4)$ of fish that could be practically handled for the tracking process, which resulted in a relatively small dataset. Therefore, we used the behavioural assessment as a hypothesis-generating tool while being careful to draw conclusions regarding the effects of NPs on zebrafish locomotive behaviour.

\section{Concluding Remarks}

Light sheet microcopy was found to be a strong tool for the in vivo screening of nano- and micro particle uptake and localisation and was demonstrated to identify target organs rapidly for NP accumulation while still preserving the integrity of the organism. By using LSM we documented NP transport via a simplified aquatic food chain, and that differential patterns for NP uptake and localisation between dietary and aqueous exposures exist. Dietary exposure to Au NPs and polystyrene NPs led to particle deposition in the stomachs and intestines of zebrafish. Conversely, after aqueous exposure, gills and intestines were identified as major uptake points. For the exploratory study on behaviour, no statistically significant effects were observed on the sensomotoric function of the zebrafish, albeit a trend of increasing relative swimming distance was observed after aqueous exposure to Au NPs after 3 and 7 days, while a trend of decreasing relative swimming distance was observed after aqueous exposure to polystyrene NPs. Dietary exposure to both types of NPs did not show any trends in the datasets. The use of light sheet microscopy enabled identification of not only (micro)plastic particle uptake by a living marine invertebrate, namely brine shrimp, from the aqueous phase, but also consequential dietary uptake in living zebrafish. The large presence of polystyrene NPs in the intestines, gills and head region should be 
considered in the context of environmental plastic pollution, as polystyrene is a widely used polymer in many consumer applications and has the potential to infiltrate aquatic ecosystems (Hammer et al., 2012; Cole et al., 2013).

\section{Acknowledgements}

The authors wish to thank the Centre for Cellular Imaging (CCI) of The Sahlgrenska Academy at Gothenburg University, Sweden, for the opportunity to use light sheet microscopy and the kind support of its staff. Lars Michael Skjolding and Anders Baun were kindly funded by ERC grant no. 281579.

\section{Declaration of interest}

This work is part of the EnvNano (Environmental Effects and Risk Evaluation of Engineered Nanoparticles) project supported by the European Research Council (Grant No. 281579). The authors are responsible for writing the article and report no conflicts of financial, consulting and personal interests. 


\section{References}

Ates, M., Arslan, Z., Demir, V., Daniels, J., Farah, I.O., 2015. Accumulation and Toxicity of CuO and $\mathrm{ZnO}$ Nanoparticles Through Waterborne and Dietary Exposure of Goldfish (Carassius auratus). Environ. Toxicol. 30, 119-128.

Asharani, P.V., Wu, Y.L., Gong, Z., Valiyaveettil, S., 2008. Toxicity of silver nanoparticles in zebrafish models. Nanotechnology 19, 255102.

Ašmonaitė, G., Boyer, S., Bresolin de Souza, K., Wassmur, B., Sturve, J., 2016, Behavioural toxicity assessment of silver ions and nanoparticles on zebrafish using a locomotion profiling approach, Aquatic Toxicology, http://dx.doi.org/10.1016/j.aquatox.2016.01.013

Batel, A., Linti, F., Scherer, M., Erdinger, L., Braunbeck, T., 2016, The transfer of benzo[a]pyrene from microplastics to Artemia nauplii and further to zebrafish via a trophic food web experiment CYP1A induction and visual tracking of persistent organic pollutants, Environ. Toxicol. Chem.

Bassi, A., Fieramonti, L., D’Andrea, C., Mione, M., \& Valentini, G, 2011, In vivo label-free threedimensional imaging of zebrafish vasculature with optical projection tomography, Journal of Biomedical Optics 16(10), 100502. doi:10.1117/1.3640808

Bondarenko, O., Juganson, K., Ivask, A., Kasemets, K., Mortimer, M., Kahru, A., 2013. Toxicity of $\mathrm{Ag}, \mathrm{CuO}$ and $\mathrm{ZnO}$ nanoparticles to selected environmentally relevant test organisms and mammalian cells in vitro: a critical review. Arch. Toxicol. 87, 1181-1200.

Cedervall, T., Hansson, L., Lard, M., Frohm, B., Linse, S., 2012. Food Chain Transport of Nanoparticles Affects Behaviour and Fat Metabolism in Fish. PLoS One 7, e32254. 
Choudhary, S., Peri, A., Dist, R. R., 2015, Advances in Alzheimer's Disease Research Using 4(3), $451-467$.

Cole, M., Lindeque, P., Fileman, E., Halsband, C., Goodhead, R., Moger, J., \& Galloway, T. S., 2013, Microplastic Ingestion by Zooplankton, Environmental Science \& Technology, 47(12), $6646-6655$.

Croteau, M., Misra, S.K., Luoma, S.N., Valsami-Jones, E., 2014. Bioaccumulation and Toxicity of $\mathrm{CuO}$ Nanoparticles by a Freshwater Invertebrate after Waterborne and Diet-borne Exposures. Environ. Sci. Technol. 48, 10929-10937.

Dai, L., Banta, G. T., Selck, H., Forbes, V. E., 2015, Influence of copper oxide nanoparticle form and shape on toxicity and bioaccumulation in the deposit feeder, Capitella teleta. Marine Environmental Research 111, 99-106.

Dedeh, A., Ciutat, A., Treguer-Delapierre, M., Bourdineaud, J.-P., 2015, Impact of gold nanoparticles on zebrafish exposed to a spiked sediment, Nanotoxicology 9(1), 71-80.

Fabrega, J., Luoma, S.N., Tyler, C.R., Galloway, T.S., Lead, J.R., 2011. Silver nanoparticles: Behaviour and effects in the aquatic environment. Environ. Int. 37, 517-531.

Geffroy, B., Ladhar, C., Cambier, S., Treguer-Delapierre, M., Brethes, D., Bourdineaud, J., 2012. Impact of dietary gold nanoparticles in zebrafish at very low contamination pressure: The role of size, concentration and exposure time. Nanotoxicology 6, 144-160.

Gerlai, R., 2011, Editorial: A small fish with a big future: Zebrafish in behavioral neuroscience: Rev. Neurosci., Reviews in the Neurosciences 22(1), 3-4. 
Hammer, J., Kraak, M.H.S., Parsons, J.R., 2012, Plastics in the Marine Environment: The Dark side of a Modern Gift, Reviews of Environmental Contamination and Toxicology.

Handy, R.D., von der Kammer, F., Lead, J.R., Hassellov, M., Owen, R., Crane, M., 2008. The ecotoxicology and chemistry of manufactured nanoparticles. Ecotoxicology 17, 287-314.

Jolck, R.I., Rydhog, J.S., Christensen, A.N., Hansen, A.E., Bruun, L.M., Schaarup-Jensen, H., von Wenck, A.S., Borresen, B., Kristensen, A.T., Clausen, M.H., Kjaer, A., Conradsen, K., Larsen, R., af Rosenschold, P.M., Andresen, T.L., 2015. Injectable Colloidal Gold for Use in Intrafractional 2D Image-Guided Radiation Therapy. Adv. Healthc. Mater. 4, 856-863.

Kalueff, A. V, Gebhardt, M., Stewart, A. M., Cachat, J. M., Brimmer, M., Chawla, J. S., Schneider, H., 2013, Towards a comprehensive catalog of zebrafish behavior 1.0 and beyond, Zebrafish 10(1), 70-86. doi:10.1089/zeb.2012.0861

Kashiwada, S., 2006. Distribution of nanoparticles in the see-through medaka (Oryzias latipes). Environ. Health Perspect. 114, 1697-1702.

Kimura, E., Isogai, S., \& Hitomi, J., 2015, Integration of vascular systems between the brain and spinal cord in zebrafish, Developmental Biology, 1-12. doi:10.1016/j.ydbio.2015.07.015

Kretzschmar, R., Holthoff, H., Sticher, H., 1998.Influence of $\mathrm{pH}$ and humic acid on coagulation kinetics of kaolinite: a dynamic light scattering study. J. Colloid Interf. Sci. 202, 95-103.

Ladhar, C., Geffroy, B., Cambier, S., Treguer-Delapierre, M., Durand, E., Brethes, D., Bourdineaud, J., 2014. Impact of dietary cadmium sulphide nanoparticles on Danio rerio zebrafish at very low contamination pressure. Nanotoxicology 8, 676-685. 
Larguinho, M., Correia, D., Diniz, M.S., Baptista, P.V., 2014. Evidence of one-way flow bioaccumulation of gold nanoparticles across two trophic levels. Journal of Nanoparticle Research $16,2549$.

Little, E. E., Archeski, R. D., Flerov, B. a., \& Kozlovskaya, V. I., 1990, Behavioral indicators of sublethal toxicity in rainbow trout, Archives of Environmental Contamination and Toxicology 19(3), 380-385. doi:10.1007/BF01054982

Ma, H., Williams, P.L., Diamond, S.A., 2013. Ecotoxicity of manufactured ZnO nanoparticles - A review. Environmental Pollution 172, 76-85.

Mattsson, K., Ekvall, M.T., Hansson, L., Linse, S., Malmendal, A., Cedervall, T., 2015. Altered Behavior, Physiology, and Metabolism in Fish Exposed to Polystyrene Nanoparticles. Environ. Sci. Technol. 49, 553-561.

Menard, A., Drobne, D., Jemec, A., 2011. Ecotoxicity of nano-sized TiO2. Review of in vivo data. Environmental Pollution 159, 677-684.

Merrifield, D. L., Shaw, B. J., Harper, G. M., Saoud, I. P., Davies, S. J., Handy, R. D., Henry, T. B., 2013, Ingestion of metal-nanoparticle contaminated food disrupts endogenous microbiota in zebrafish (Danio rerio), Environmental Pollution 174, 157-163.

Ramsden, C.S., Smith, T.J., Shaw, B.J., Handy, R.D., 2009. Dietary exposure to titanium dioxide nanoparticles in rainbow trout, (Oncorhynchus mykiss): No effect on growth, but subtle biochemical disturbances in the brain. Ecotoxicology 18, 939-951. 
Ramskov, T., Thit, A., Croteau, M.-N., \& Selck, H., 2015, Biodynamics of copper oxide nanoparticles and copper ions in an oligochaete - Part I: Relative importance of water and sediment as exposure routes, Aquatic Toxicology, 164, 81-91

Scown, T.M., van Aerle, R., Tyler, C.R., 2010. Review: Do engineered nanoparticles pose a significant threat to the aquatic environment? Crit. Rev. Toxicol. 40, 653-670.

Scott, G. R., \& Sloman, K. A., 2004, The effects of environmental pollutants on complex fish behaviour: Integrating behavioural and physiological indicators of toxicity, Aquatic Toxicology 68(4), 369-392. doi:10.1016/j.aquatox.2004.03.016

Shaw, B.J., Handy, R.D., 2011. Physiological effects of nanoparticles on fish: A comparison of nanometals versus metal ions. Environ. Int. 37, 1083-1097.

Skjolding, L.M., Winther-Nielsen, M., Baun, A., 2014. Trophic transfer of differently functionalized zinc oxide nanoparticles from crustaceans (Daphnia magna) to zebrafish (Danio rerio). Aquatic Toxicology 157, 101-108.

Tourinho, P.S., van Gestel, C.A.M., Lofts, S., Svendsen, C., Soares, A.M.V.M., Loureiro, S., 2012. Metal-based nanoparticles in soil: Fate, behavior, and effects on soil invertebrates. Environmental Toxicology and Chemistry 31, 1679-1692.

Truong, L., Saili, K. S., Miller, J. M., Hutchison, J. E., \& Tanguay, R. L., 2012, Persistent adult zebrafish behavioral deficits results from acute embryonic exposure to gold nanoparticles. Comparative Biochemistry and Physiology. Toxicology \& Pharmacology : CBP, 155(2), 269-74. 
White, R. M., Sessa, A., Burke, C., Bowman, T., LeBlanc, J., Ceol, C., Zon, L. I., 2008, Transparent Adult Zebrafish as a Tool for In Vivo Transplantation Analysis, Cell Stem Cell 2(2), 183-189. doi:10.1016/j.stem.2007.11.002

Xin Q., Zhang Q., Cheng J., 2014. Review on the Toxicology Study of Silver Nanoparticles on Fish Species. Asian Journal of Ecotoxicology 9, 1014-1026.

Zhu, Z., Carboni, R., Quercio, M.J., Jr., Yan, B., Miranda, O.R., Anderton, D.L., Arcaro, K.F., Rotello, V.M., Vachet, R.W., 2010. Surface Properties Dictate Uptake, Distribution, Excretion, and Toxicity of Nanoparticles in Fish. Small 6, 2261-2265.

\section{Figures and Table Legends}

Figure 1: Maximum-intensity projection of light sheet microscopy zebrafish images exposed to fluorescent polystyrene NPs for 1, 3 and 7 days through the aqueous phase (a, b and c), and preexposed brine shrimp ( $\mathrm{d}$, e and $\mathrm{f}$ ) as diet, respectively. $\mathrm{G}$ is a transmission image of a control fish for orientation. Red signal corresponds to fluorescent polystyrene nanoparticles, and the grey signal corresponds to the autofluorescence of the zebrafish. OR: Olfactory region, GI: Gills, PF: Pectoral fin and GU: Gut.

Figure 2: Maximum-intensity projection of light sheet microscopy zebrafish images exposed to $1 \mathrm{mg}$ $\mathrm{Au} / \mathrm{L}$ fluorescent $\mathrm{Au} \mathrm{NP}$ functionalised with an fluorescein probe at $0.5 \%$ for 1,3 and 7 days through the aqueous phase ( $a, b$ and $c$ ) and through diet (d, e and f) with brine shrimp pre-exposed to $1 \mathrm{mg} \mathrm{Au} / \mathrm{L} \mathrm{Au} \mathrm{NP}$ functionalised with an fluorescein probe at $0.5 \%$. $\mathrm{G}$ is a transmission image of a control fish for orientation. The bright white signal corresponds to fluorescent $\mathrm{Au} \mathrm{NP}$, and the grey signal corresponds to the autofluorescence of the zebrafish. OR: Olfactory region, GI: Gills, PF: Pectoral fin and GU: Gut. 
Figure 3: Mass quantification of Au uptake in zebrafish during a seven-day exposure to Au NPs through the aqueous phase (a) at $1 \mathrm{mg} \mathrm{Au} / \mathrm{L}$ and dietary exposure (b) with brine shrimp having a body burden of ng $27 \pm 3.1 \mathrm{ng} \mathrm{Au} / \mu \mathrm{g}$ dry weight. Data presented as means $\pm \mathrm{SD}$.

Figure 4: Relative swimming distance of Au NP- (a) and polystyrene NP- (b) exposed zebrafish via dietary and aqueous routes, normalised to the control group: $n=4$, data presented as means \pm SE.

Table 1: Characterisation of fluorescent Au NPs and polystyrene NPs in zebrafish medium, using DLS to determine size (z-average), the polydispersity index (PDI) and stability ( $\zeta$-potential) after $10 \mathrm{~min}$ and $24 \mathrm{~h}$ of incubation. 
Figures

Fig 1:

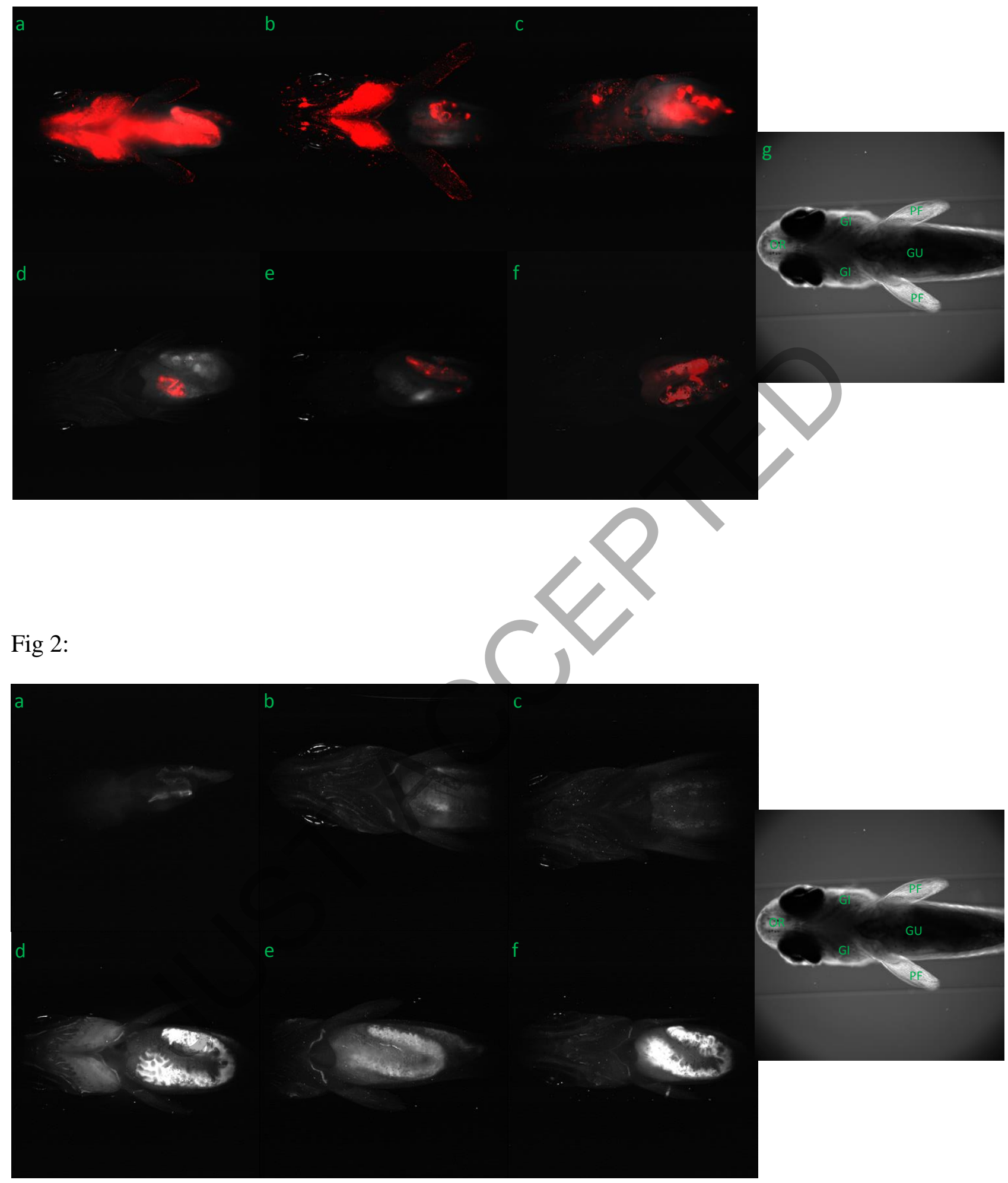


Fig 3:

a)
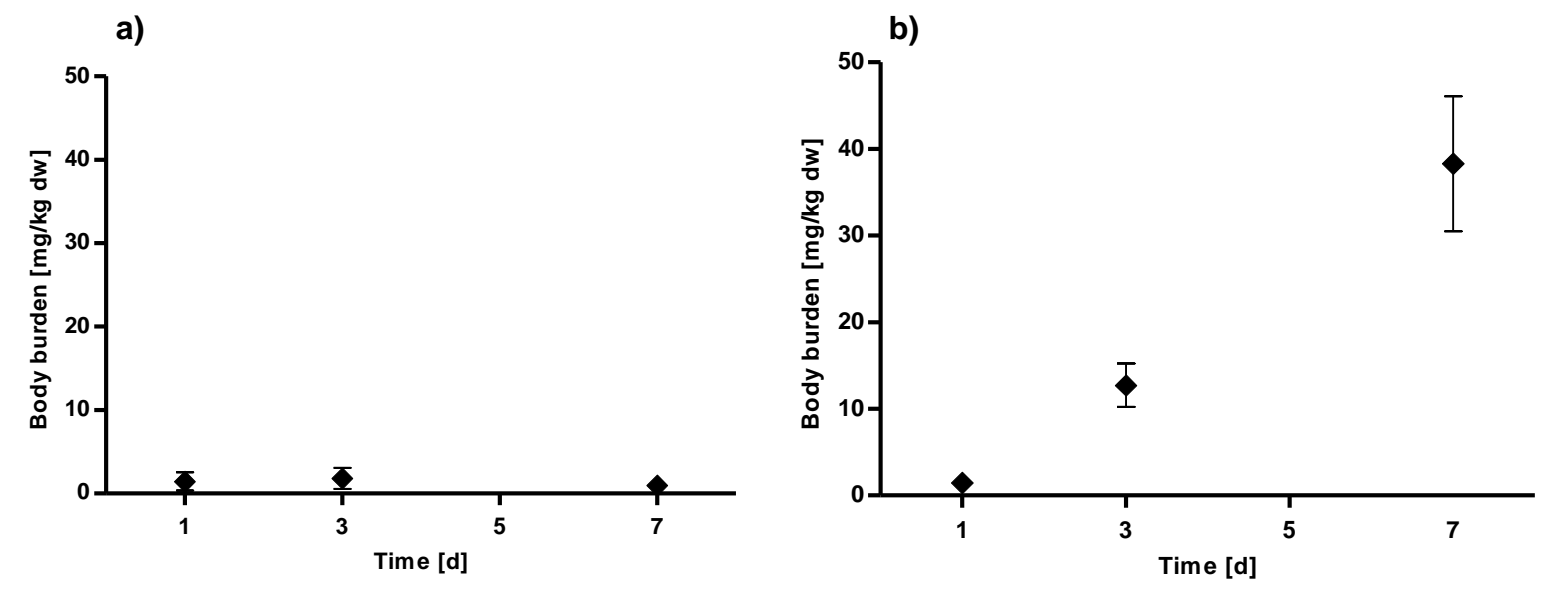

Fig 4:

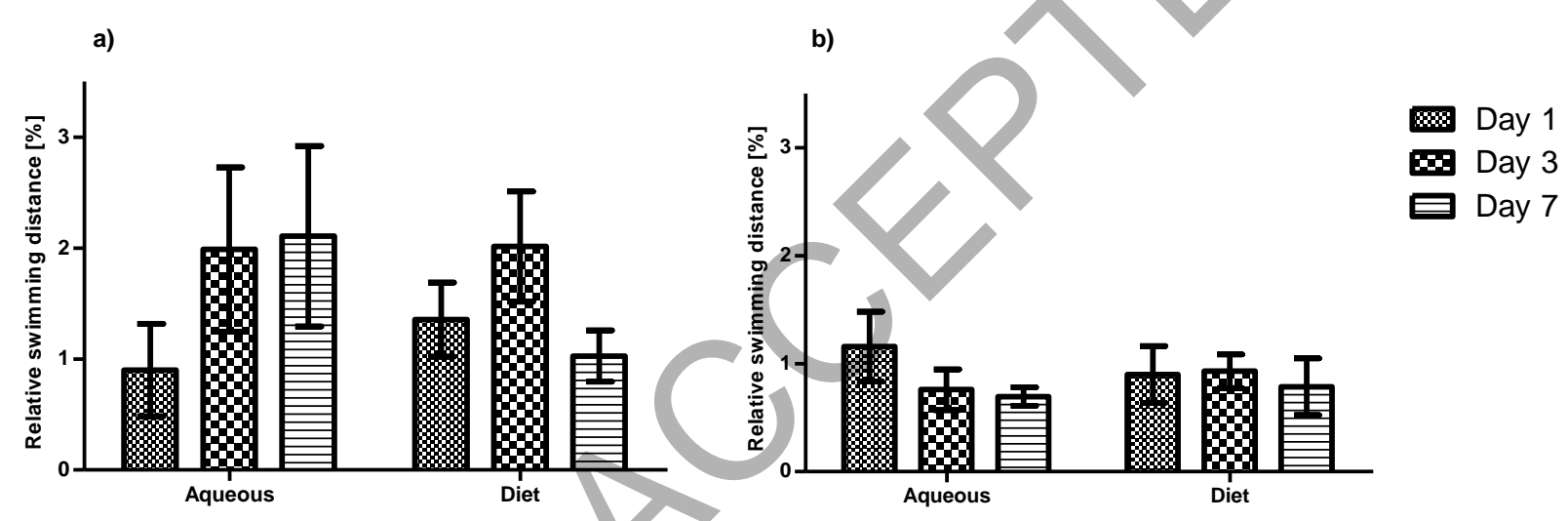




\section{Tables}

Table 1:

\begin{tabular}{|c|c|c|c|c|c|c|}
\hline \multirow{3}{*}{ Test NPs } & \multirow{2}{*}{\multicolumn{2}{|c|}{$\begin{array}{c}\text { Z-average } \\
{[\mathrm{nm}]}\end{array}$}} & \multirow{2}{*}{\multicolumn{2}{|c|}{$\begin{array}{l}\text { PDI* }^{*} \\
{[\mathrm{~nm}]}\end{array}$}} & \multirow{2}{*}{\multicolumn{2}{|c|}{$\begin{array}{c}\zeta \text {-potential } \\
{[\mathrm{mV}]}\end{array}$}} \\
\hline & & & & & & \\
\hline & $\mathrm{t}=10 \mathrm{~m}$ & $\mathrm{t}=24 \mathrm{~h}$ & $\mathrm{t}=10 \mathrm{~m}$ & $t=24 h$ & $\mathrm{t}=10 \mathrm{~m}$ & $t=24 h$ \\
\hline Au NPs & $71 \pm 4$ & $89 \pm 21$ & $0.21 \pm 0.08$ & $0.27 \pm 0.03$ & $-22.2 \pm 2$ & $-20 \pm 0.3$ \\
\hline Polystyrene NPs & $610 \pm 130$ & $3580 \pm 410$ & $0.44 \pm 0.05$ & 1 & $-13.4 \pm 0.4$ & $-13.5 \pm 0.3$ \\
\hline
\end{tabular}

* For poly-disperse samples (PDI > 0.2), data should only be used to interpret trends in data. 